\title{
Attenuation of Hydromagnetic Waves in the Ionosphere
}

\author{
Syun-Ichi Akasofu
}

\author{
Contribution From the Geophysical Institute, University of Alaska, College, Alaska
}

(Received October 20, 1964)

\begin{abstract}
Effects of the daily variation of the ionosphere on the attenuation of hydromagnetic waves are examined. The ionosphere just before sunrise is almost transparent to the extraordinary component of the waves of frequencies between $10^{-3}$ and $10^{2} \mathrm{c} / \mathrm{s}$, but becomes fairly opaque during daylight hours. An intense ionization in the $E$ region, namely, the sporadic $E$, can heavily attenuate the waves of frequencies above $10^{-2} \mathrm{c} / \mathrm{s}$. It is concluded that the daily, latitudinal, and seasonal variations of the ionosphere must carefully be taken into account in order to examine characteristics of the source of geomagnetic micropulsations.
\end{abstract}

\section{Introduction}

The propagation of hydromagnetic waves in an infinitely conductive fluid was studied first by Alfvén [1950]. It was then demonstrated by Aström [1950]; Schlüter [1952]; Dungey [1954a]; Ferraro [1955], and others that hydromagnetic waves can propagate in a fully ionized gas, provided that frequencies of the wave are well below the gyrofrequency of positive ions.

Hines [1953] was the first who examined the propagation of hydromagnetic waves in a gas of incomplete ionization. He showed that the problem can be treated as an extension of the well-known magnetoionic theory to a very-low-frequency range such that motions of positive ions and even neutral particles must be taken into account. In the magneto-ionic theory, only electrons respond to electromagnetic waves in an immobile background, namely the positive ions and neutral particles. These theoretical studies led Dungey $[1954 \mathrm{~b}$, c] to suggest that some of the geomagnetic micropulsations are of hydromagnetic origin. A large number of works have then been devoted to understand the nature and origin of geomagnetic micropulsations in terms of hydromagnetic waves.

Figure 1 shows typical examples of micropulsations recorded at College, Alaska (gm. lat. 64.5 $\mathrm{N}$ ). Geomagnetic micropulsations appear in a wide frequency range. The top example is the so-called "pearl-type" micropulsation (recorded by the earth current meter). Such regular sinusoidal oscillations appear in periods between 0.3 and 4 sec, but most commonly 1 sec [Troitskaya, 1964]. The middle example is also a pearl-type micropulsation with the period of order 4 sec. The bottom example is the so-called "giant pulsation" which attracted the attention of many of the early workers in the field of geomagnetism [Rolf, 1931; Harang, 1936 and 1939; Sucksdorff, 1936]. One of the most characteristic features of some of the geomagnetic micropulsations is a sort of amplitude
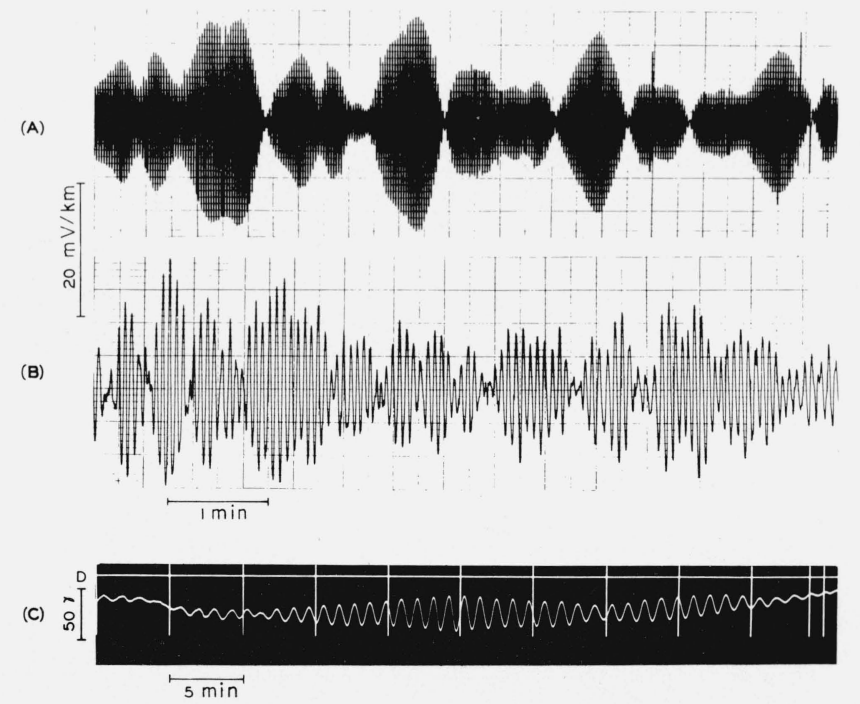

FIGURE 1. Examples of geomagnetic micropulsations recorded at College, Alaska; (A) the pearl-type telluric current micropulsations of period of order of 1 sec., $(B)$ the pearl-type telluric current micropulsations of period of order 4 sec., $(C)$ the giant pulsation (the $D$ component) recorded by the College rapid-run magnetometer. 
modulation when they are shown in an amplitudetime record. Tepley and Wentworth [1962] showed by use of a sonagram technique that this amplitude modulation pattern in the amplitude-time record is produced by a series of overlapping wave trains of rising frequencies.

We know at present very little about the origin of geomagnetic micropulsations, except some of them generated apparently at the time of a sudden impact of intense solar plasma flow on the magnetosphere [Wilson and Sugiura, 1961]. One can reasonably infer that there must be fairly stable "oscillators" to generate such geomagnetic micropulsations which we now identify as hydromagnetic waves. The "oscillators" must be extremely stable; in an amplitudetime display, the periods of the pearl-type micropulsations are greatly concentrated at about $1 \mathrm{sec}$, and giant pulsations can last for almost 1 hour without changing period significantly (see fig. 1).

Such a remarkable stability has led one to infer that the wave frequencies are closely related to some basic physical quantities or to some stable geometrics or to both [cf. Dungey, 1954b, c; Wentworth and Tepley 1962; Jacobs and Watanabe 1963]. Unfortunately, we are still far from a reasonable solution to the problem; however, it seems very likely that most of such hydromagnetic waves are generated in the region beyond the ionosphere. As is clear from early studies [Hines 1953; Piddington 1956; Wantanabe 1957; Dessler 1959; Fejer 1960; Akasofu 1960; Francis and Karplus 1960; Karplus et al., 1962], the ionosphere acts as a sort of filter to such waves.

The ionosphere undergoes various changes including the daily, seasonal, storm-time variations. Thus, we record the waves on the ground through a filter of varying characteristics. The daily variation of characteristics of geomagnetic micropulsations recorded on the ground has been supposed to give some clue on their origin or excitation mechanisms. It is obvious, however, that the filtering effects of the ionosphere on the waves must be removed from records to obtain a better understanding of their daily characteristics. In this paper, the attentuation of hydromagnetic waves is examined for various ionospheric models, with particular attention to the daily variation of the ionosphere.

\section{Model Ionospheres}

Our main purpose here is to examine how various levels of the ionosphere affect hydromagnetic waves through their daily variation. Figure 2 shows the model ionsopheres used in this paper. The number indicated in each curve refers to the local time. The model examined by Prince, Bostick, and Smith [1964] is used as a typical noon profile (12) of the electron density (their table 1). In the region between 90 and 200 $\mathrm{km}$, the number of density of the electrons changes approximately with $\sqrt{\cos \chi}$ (where $\chi$ denotes the solar zenith angle) at about the geographic latitude of $45^{\circ}$. The change of the electron density beyond $200 \mathrm{~km}$ cannot be expressed in any simple way, so that the profiles are constructed by use of typical ionograms.

In addition to the above daily change, we examine effects of the sporadic $E$ layer $(E s)$. For this purpose, the electron density profile at midnight $(0)$ is modified below $200 \mathrm{~km}$ to give a region of intense ionization centered at $110 \mathrm{~km}$. This modified profile is referred to as "A".

In the above daily and sporadic variations of the ionosphere, it is assumed that the number density of the neutral atoms in the ionosphere remains constant. It has recently become clear that there exists an appreciable daily variation of the number density in the ionospheric region. In order to see an effect of the change in number density of neutral atoms on the

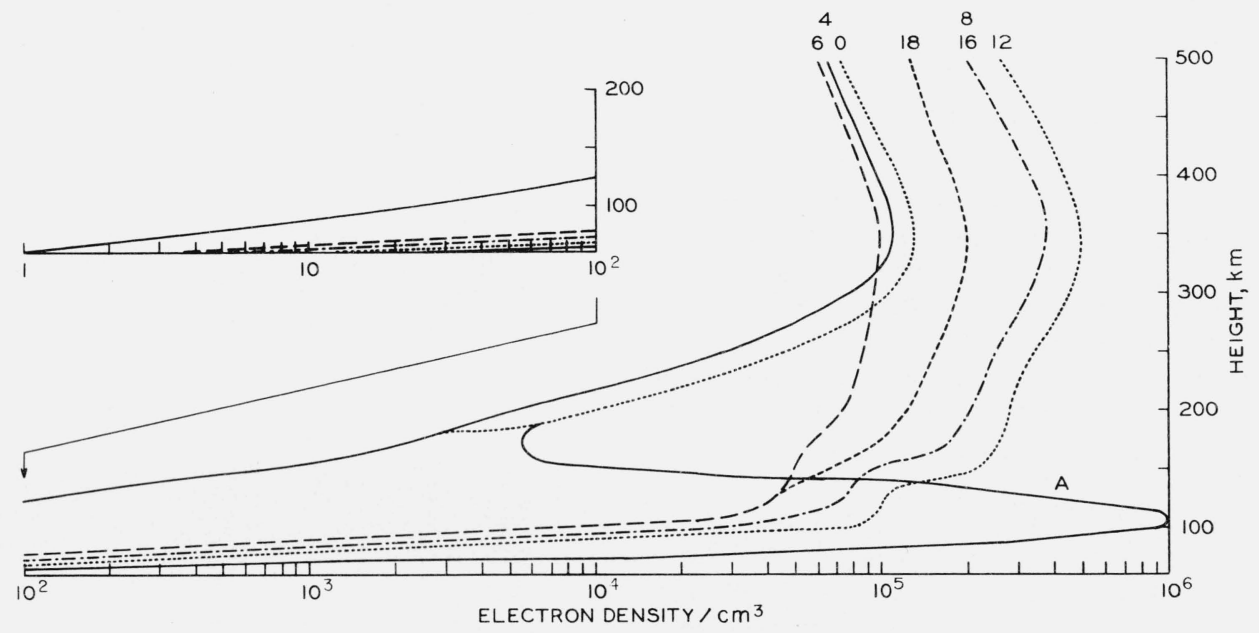

FIGURE 2. The model ionospheres used for the calculation of the attenuation of hydromagnetic waves; the numbers indicated in each curve refers to the local time; for example " 0 " refers to the local midnight; the curve $B$ is taken as a model sporadic E layer. 
FREQUENCY $=1 \mathrm{c} / \mathrm{s}$

ORDINARY COMP. ......-

EXTRA. O. COMP.

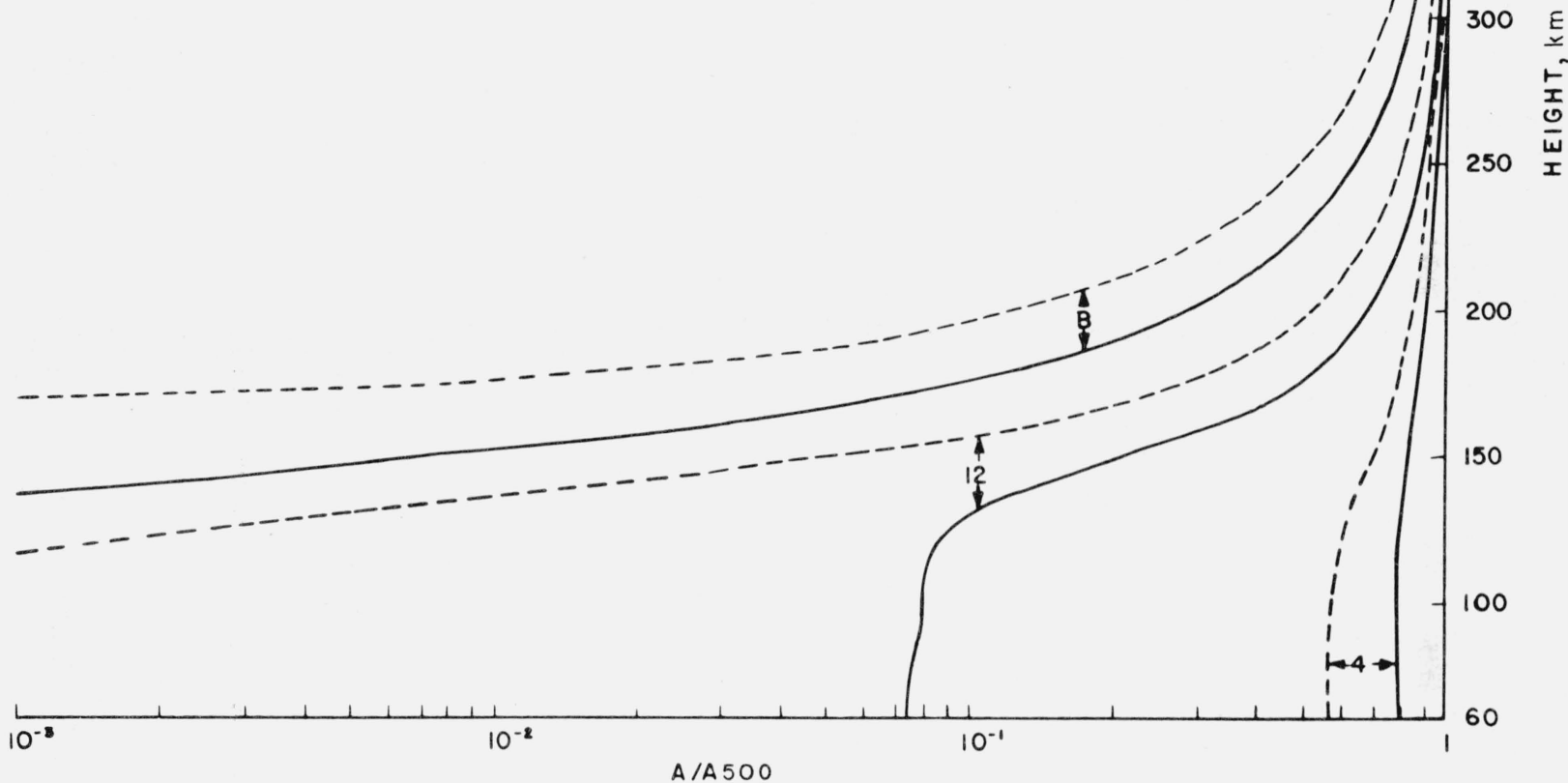

Figure 3. The ratio $\mathbf{A} / \mathbf{A}_{500}$ as a function of height for hydromagnetic waves of period 1 sec. (or frequency $1 \mathrm{c} / \mathrm{s}$ ) for the model ionospheres at 4 a.m. (4), noon (12) and B, respectively. If the amplitude of the waves is taken to be one gamma at $500 \mathrm{~km}$, the abscissa $\left(\mathrm{A} / \mathrm{A}_{500}\right)$ gives numerically the amplitude at a given height in the ordinate.

attenuation of hydromagnetic waves, the number density of the neutral atoms at noon is simply increased tenfold, although such a large daily change is unlikely. The ratio of the number density of neutral particles at the period of sunspot maximum to that at the period of sunspot minimum may, however, be of this order. This particular model ionosphere is referred to as "B".

\section{Calculation of the Attenuation}

In order to simplify the problem, plane hydromagnetic waves of the form $\exp i(\omega t+\kappa z)$ are assumed to propagate along the geomagnetic field lines (along the $z$-axis in a Cartesian coordinate system) permeating the ionosphere vertically. Hines' general equation of dispersion [his eq (19)]

$$
\begin{aligned}
& \kappa^{2}-\omega^{2} \epsilon \mu=-\omega \mu \Sigma \frac{N_{r} e_{r}^{2}}{M_{r}\left(\omega \pm G_{r}-i K_{r}\right)} \\
&+\frac{\omega \mu\left(\Sigma \frac{N_{r} e_{r} K_{r}}{\omega \pm G_{r}-i K_{r}}\right)^{2}}{\omega \rho-i \Sigma \frac{N_{r} M_{r} K_{r}\left(\omega \pm G_{r}\right)}{\omega \pm G_{r}-i K_{r}}}
\end{aligned}
$$

is used to calculate the complex wave number which may be written as

$$
\kappa=\alpha+i \beta
$$

where $\alpha$ and $\beta$ are real and positive numbers.

Here, $M_{r}, N_{r}$, and $e_{r}$ are the mass and number density and charge of the $r$ th particle, respectively, and $K_{r}$ and $G_{r}$ the collision [Hines, 1953 eq (3)] and the 
gyrofrequency, respectively. The ionosphere may be treated as a ternary mixture composed of electrons and positive ions, together with neutral particles; it is assumed that the mass $M$ of both ions and neutral particles is the same and equal to the mean molecular mass. The mass density $M N$ of neutral atoms is denoted by $\rho$ in the dispersion equation.

In the actual calculation, the ionosphere is approximated by a number of slabs of thickness $\delta=10 \mathrm{~km}$ in which all the quantities are uniform. Therefore, the complex wave number can be calculated in each slab. Since most of the attenuation takes place below 500 $\mathrm{km}$ in altitude, the amount of attenuation from the top of the ionosphere to a particular level of the ionosphere at height $h$ may be given by the ratio of amplitude $A$ of hydromagnetic waves at the particular level to that $\left(A_{500}\right)$ at a $500 \mathrm{~km}$ level, namely $A / A_{500}$. It is given by

$$
\frac{A_{(h)}}{A_{500}}=\exp \left\{-\int_{500}^{h} \beta(z) \delta d z\right\} \text {. }
$$

Figure 3 shows the distribution of $A / A_{500}$ as a function of height for the waves of period $1 \mathrm{sec}$ (or frequency $1 \mathrm{c} / \mathrm{s}$ ). As we shall see shortly, as far as the attenuation is concerned, the ionospheres at noon (12) and at 4 a.m. (4) can be taken to be the two extreme cases, the minimum occurring at 4 a.m. (just before sunrise at $200 \mathrm{~km}$ level) and the maximum, at noon.

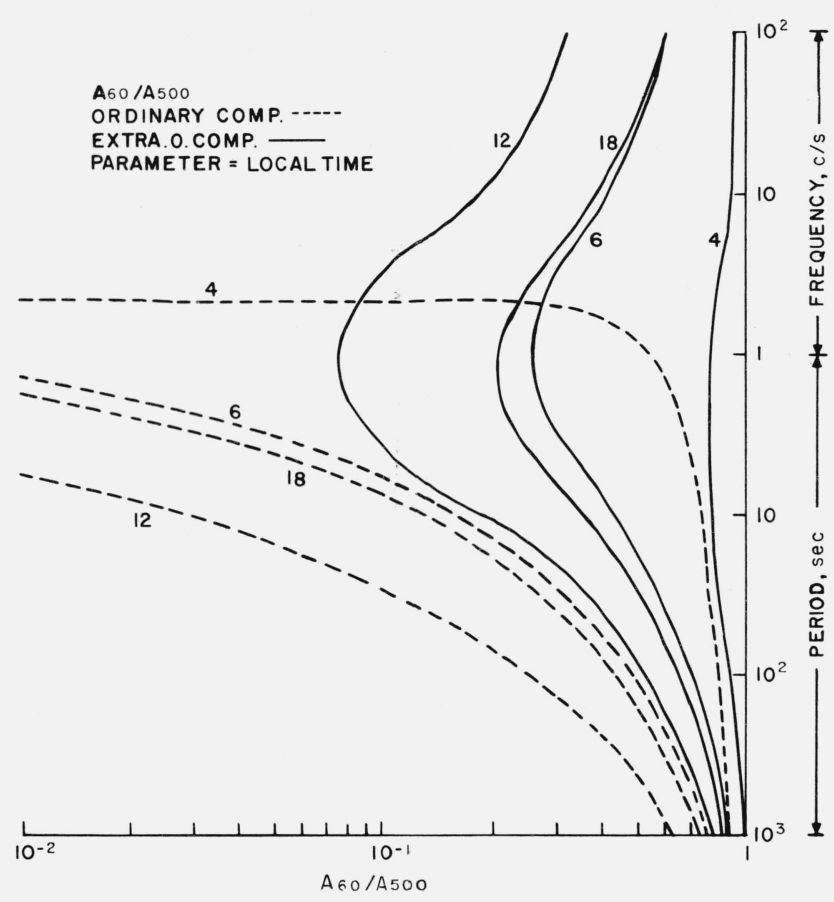

FIGURE 4. The ratio $\mathrm{A}_{60} / \mathrm{A}_{500}$ as a function of period and frequency.

The abscissa $\left(A_{60} / A_{500}\right)$ gives numerically the amplitude of hydromagnetic waves at a 60 $\mathrm{km}$ level for different periods and frequencies (the ordinate) if the amplitude is taken to be $\mathrm{km}$ level for different periods and frequencies (the ordinate if the amplitude is taken to be
one gamma at a $500 \mathrm{~km}$ level. The number indicated in each curve refers to the local time.

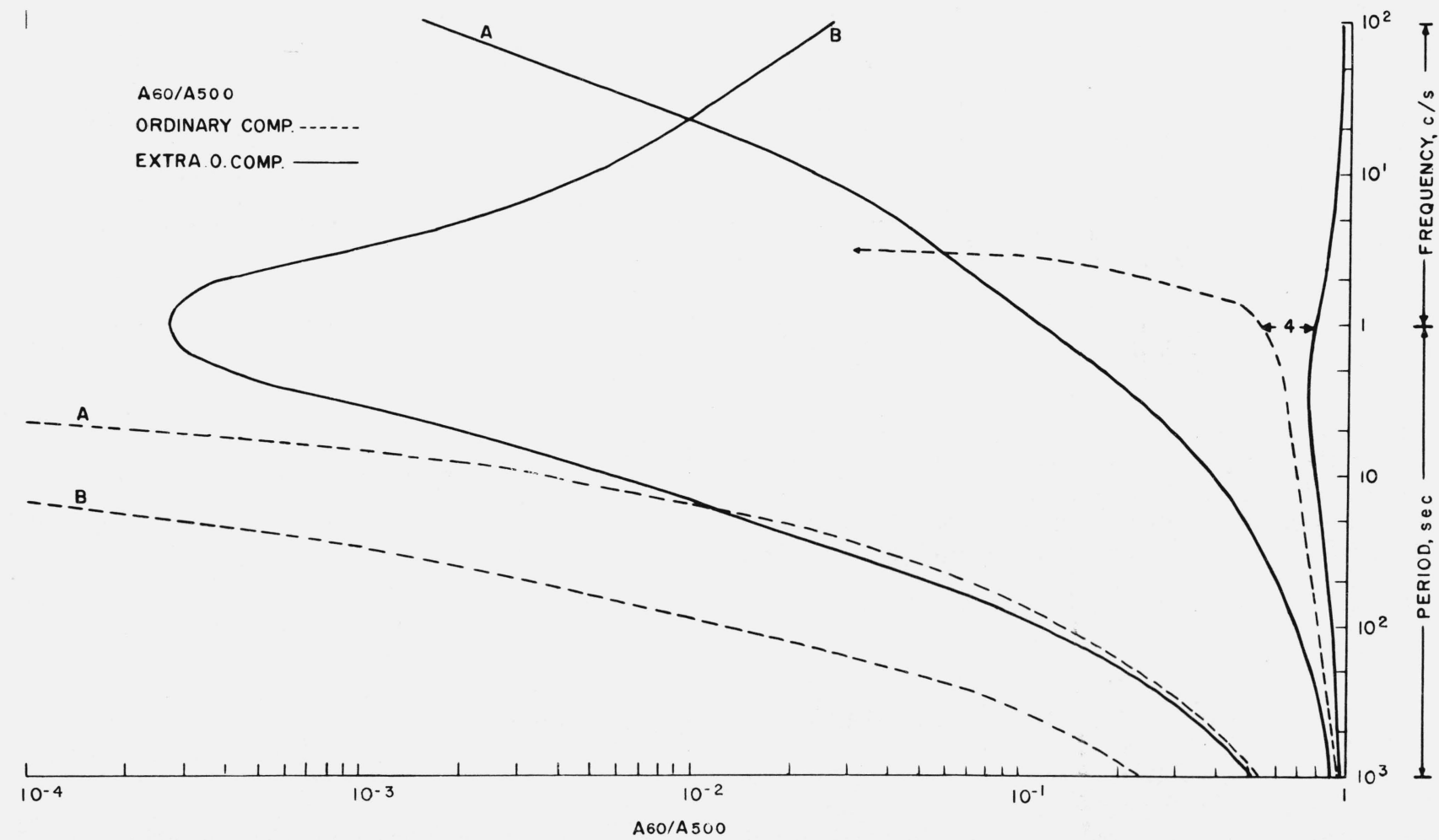

FIGURE 5. The ratio $\mathrm{A}_{60} / \mathrm{A}_{500}$ as a function of period and frequency for the model ionospheres, 4, $A$ and $B$. For details, see the caption for figure 4 and the text. 
First of all, it is clear that the attenuation takes place mostly below $250 \mathrm{~km}$ in altitude, both at noon and $4 \mathrm{a} . \mathrm{m}$. The ionization in the $F^{2}$ region does not seriously affect the attenuation. Wentworth [1964] took the $F^{2}$ peak ion density as an index to examine the ionospheric attenuation of micropulsations; the present calculation shows that it may not be a good index to choose. Second, we note that at 4 a.m. a substantial portion of the wave energy for both the ordinary and extraordinary waves can penetrate through the ionosphere; this is because the electron density below $250 \mathrm{~km}$ at $4 \mathrm{a} \cdot \mathrm{m}$. is not large enough to seriously affect the wave propagation. It may be inferred that this situation is also close enough for the polar ionosphere in winter. Dr. J. H. Piddington, C.S.I.R.O., has suggested this to me. Third, at noon, the ordinary component of the wave is far more seriously attenuated than the extraordinary component. Below $60 \mathrm{~km}$, the attenuation becomes almost negligible.

The curve $B$ suggests that if the number density of the neutral particles is increased by tenfold, both components of the wave can be very seriously attenuated. Therefore, if there exists an increase of the number density during daylight hours, the attenuation shown by the curve 12 may be increased. The 11-yr. cycle variation of the occurrence frequency of some of the geomagnetic micropulsation may be due partly to the change of the ionization below $250 \mathrm{~km}$ and partly to a large increase of the number density of the neutral particles [see also Karplus et al., 1962].

The ratio of the amplitude of the waves at $60 \mathrm{~km}$ to that at $500 \mathrm{~km}$, namely $A_{60} / A_{500}$, will give a fairly accurate measure of the attenuation of hydromagnetic waves through the ionosphere. Figure 4 shows $A_{60} / A_{500}$ as a function of the period (sec) and frequency (c/s) of the waves. For the ordinary component, the attenuation increases steadily with decreasing period (or increasing frequency). The extraordinary component shows, however, a little maximum of the absorption at about a period of 1 sec; the attenuation then decreases with increasing frequency to about $100 \mathrm{c} / \mathrm{s}$. Above this frequency the attenuation increases steadily, although this is not shown in the figure. The slight increase of the attenuation around the period of $1 \mathrm{sec}$ is not a universal phenomenon (see below).

The ionosphere is essentially transparent to the extraordinary component of hydromagnetic waves of periods between $10^{3} \mathrm{sec}$ and $10^{-2} \mathrm{sec}$ (or of frequencies between $10^{-3} \mathrm{c} / \mathrm{s}$ and $10^{2} \mathrm{c} / \mathrm{s}$ ) at $4 \mathrm{a} . \mathrm{m}$. or in the polar winter condition. The spectrum of the extraordinary component of the waves obtained on the ground in the early morning may be essentially the same as that above the ionosphere. However, we cannot expect the ordinary component of the waves of frequency above $20 \mathrm{c} / \mathrm{s}$ in any circumstance. Figure 5 shows also $A_{60} / A_{500}$ as a function of period or frequency for the models $A$ and $B$, together with the curve 4 for comparison. A heavy ionization in the $E$ region can drastically alter the attenuation condition (see the curve A). The effect is more serious in the higher frequency range, but not serious for the waves of periods more than $10^{3}$ sec. This situation may be particularly important in the auroral zone where an intense bombardment of the ionosphere by energetic particles occurs [cf. Heacock and Hessler, 1962; Campbell and Leinbach 1961]. Curve B is already discussed in connection with figure 2.

Figure 6 shows the calculated daily variation of $A_{60} / A_{500}$ for the waves of periods between $1 \mathrm{sec}$ and $10^{3}$ sec. The attenuation of the waves during daylight

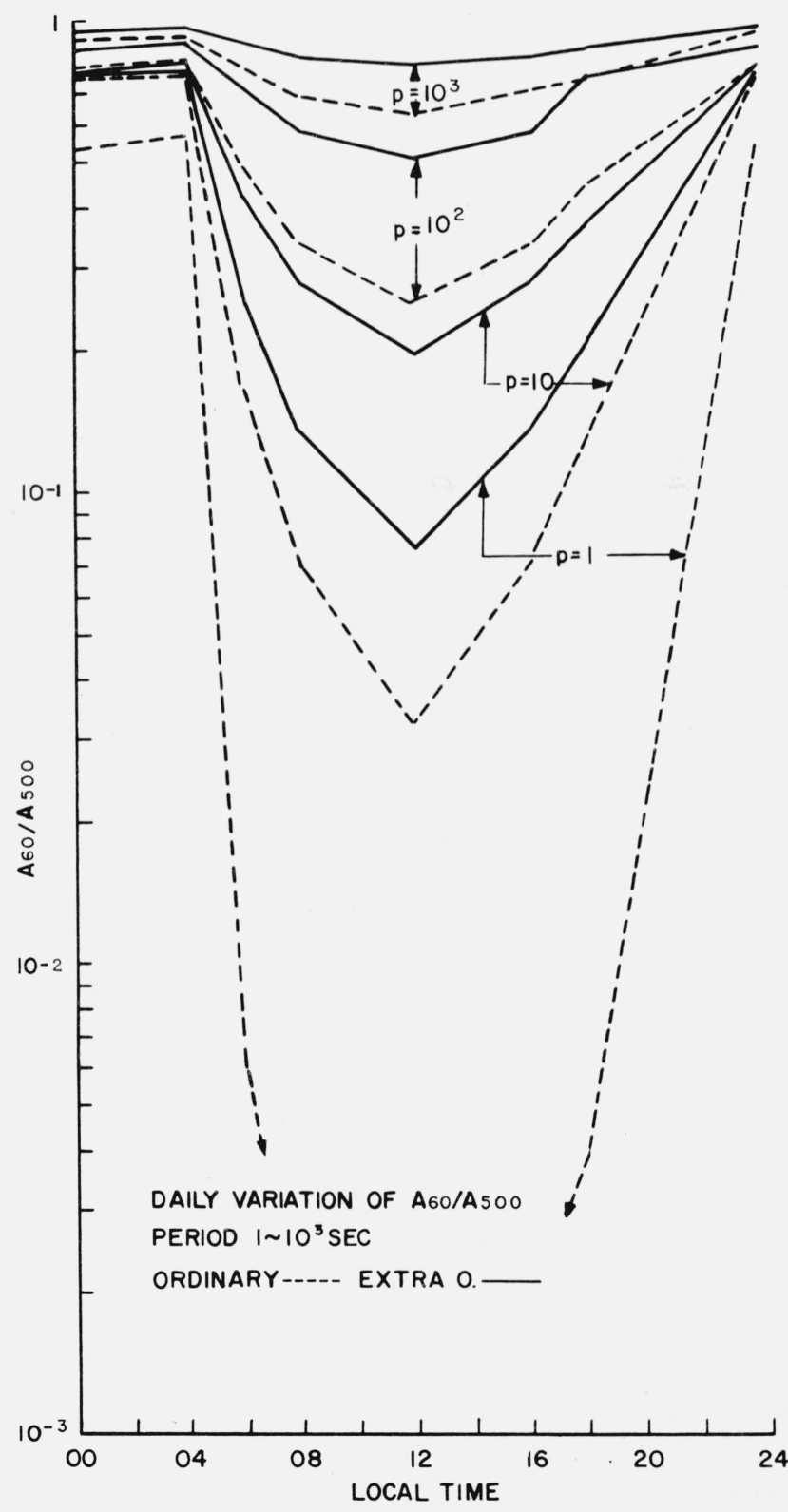

FIGURE 6. The calculated daily variation of the amplitude of hydromagnetic waves with periods between 1 and $10^{3} \mathrm{sec}$.

If the amplitude of the waves at a $500 \mathrm{~km}$ level remains constant, throughout a day, $A_{60} / A_{500}$ gives numerically the expected daily variation of the amplitude due to changes of the ionosphere during the course of a day. 


\section{References}

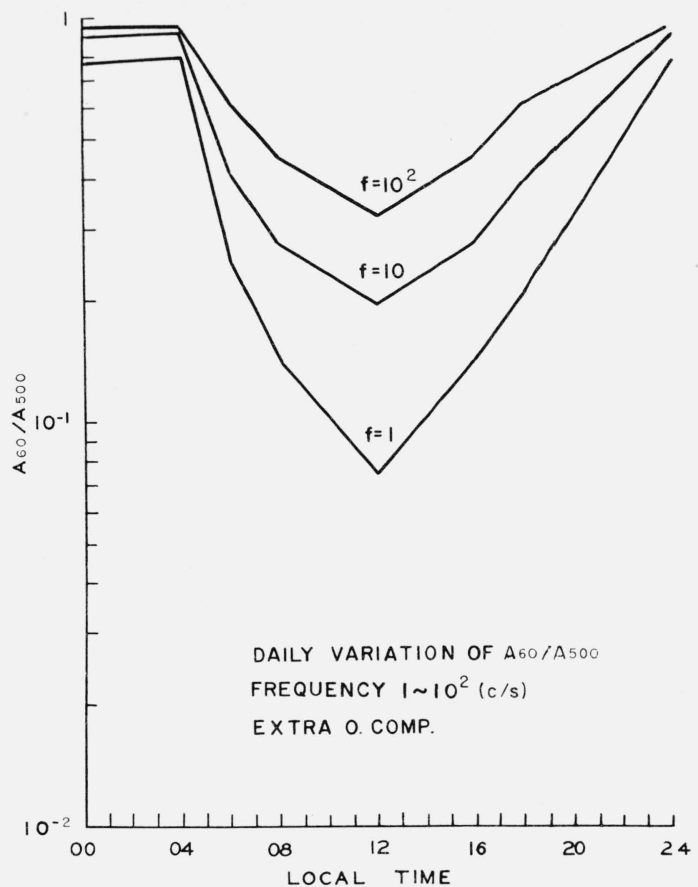

FIGURE 7. The calculated daily variation of the amplitude of hydromagnetic waves with frequencies between 1 and $10^{2} \mathrm{c} / \mathrm{s}$. For details, see the caption for figure 6 .

hours is clearly seen over the entire period, particularly around the period of 1 sec. Figure 7 shows a similar diagram for the frequency range between $1 \mathrm{c} / \mathrm{s}$ and $10^{2} \mathrm{c} / \mathrm{s}$.

\section{Discussion}

It is clear that the daily variation of the ionosphere affects greatly the daily characteristics of geomagnetic micropulsations observed on the ground. The ionosphere behaves differently at different latitudes and in different seasons. These effects must carefully be taken into account, when the latitudinal and seasonal variations of geomagnetic micropulsations are discussed.

I express my thanks to Mrs. Jean Chorbajian for her help in the computation contained in this paper. I would also thank V. P. Hessler and R. Heacock for their kind discussions and for providing telluric current records in figure 1 . These records were taken under a project supported by Air Force Cambridge Research Laboratories, Office of Aerospace Research Contract No. AF 19(628)-1695. The research reported here was in part supported by a grant from the National Aeronautics and Space Administration No. NsG 201-62.
Akasofu, S-I. (1960), On the ionospheric heating by hydromagnetic waves connected with geomagnetic micropulsations, J. Atmospheric Terrest. Phys. 18, 160-173.

Alvén, H. (1950), Cosmical electrodynamics (Oxford University Press, Oxford, England).

Aström, E. (1950), On waves in an ionized gas, Arkiv Fysik 2, 443457.

Campbell, W. H., and H. Leinbach (1961), Ionospheric absorption at times of auroral and magnetic pulsations, J. Geophys. Res. 66, $25-34$.

Dessler, A. J. (1959), Ionospheric heating by hydromagnetic waves, J. Geophys. Res. 64, 379-401.

Dungey, J. W. (1954a), The propagation of Alvén waves through the ionosphere, Ionosphere Res. Lab. The Pennsylvania State University Sci. Report No. 57.

Dungey, J. W. (1954b), The attenuation of Alvén waves, J. Geophys. Res. 50, 323-328.

Dungey, J. W. (1954c), Electrodynamics of the outer atmosphere, Ionosphere Res. Lab. The Pennsylvania State University Sci. Report No. 69.

Fejer, J. A. (1960), Hydromagnetic wave propagation in the ionosphere, J. Atmospheric Terrest. Phys. 18, 135-146.

Ferraro, V. C. A. (1955), Hydromagnetic waves in a rare ionized gas and galatic magnetic fields, Proc. Roy. Soc. A 233, 310-318.

Francis, W. E., and R. Karplus (1960), Hydromagnetic waves in the ionosphere, J. Geophys. Res. 65, 3593-3600.

Harang, L. (1936), Oscillations and vibrations in magnetic records at high-latitude stations, Terrest. Magnet. Atmosphere Elect. 41, 329-336.

Harang, L. (1939), Pulsations in an ionized region at height of 650$800 \mathrm{~km}$ during the appearance of giant pulsations in the geomagnetic records, Terrest. Magnet. Atmospheric Elect. 44, 17-19.

Heacock, R. R., and V. P. Hessler (1962), Pearl-type telluric current micropulsations at College, J. Geophys. Res. 67, 3985-3996.

Hines, C. O. (1953), Generalized magnetohydrodynamic formula, Proc. Cambridge Phil. Soc. 49, 299-307.

Jacobs, J. A., and T. Watanabe (1963), Trapped charged particles as the origin of short period geomagnetic pulsations, Planetary Space Sci. 11, 869-878.

Karplus, R., W. E. Francis, and A. J. Dragt (1962), The attenuation of hydromagnetic waves in the ionosphere, Planetary Space Sci. 9, 771-786.

Piddington, J. H. (1956), Solar atmospheric heating by hydromagnetic waves, Monthly Notices Roy. Astron. Soc. 116, 314-323.

Prince, C. E., F. Z. Bostick, Jr., and H. W. Smith (1964), A study of the transmission of plane hydromagnetic waves through the upper atmosphere, Electrical Engineering Res. Lab. University of Texas Report No. 134.

Rolf, B. (1931), Giant micropulsations at Abisko, Terrest. Magnet. Atmospheric Elect. 36, 9-14.

Schlüter, A. (1952), Der Zusammenhang der Schwingungsform eines plasma, Aum. de Phys. 10, 418-412.

Sucksdorff, E (1936), Occurrence of rapid micropulsations at Sodankylä during 1932 to 1935 , Terrest. Magnet. Atmospheric Elect. 41, 337-344.

Tepley, L. R., and R. C. Wentworth (1962), Hydromagnetic emissions, X-ray burst, and electron bunches, 1. Experimental results. J. Geophys. Res. 67, 3317-3343.

Troitskaya, V. A. (1964), Rapid variations of the electromagnetic field of the earth, Res. Geophysics 1, 485-532, ed. H. Odishaw, MIT Press.

Watanabe, T. (1957), Electrodynamic behavior and screening effect of the ionosphere, Sci. Report, Tohoku University, Geophys. 9, 81-98.

Wentworth, R. C., and L. R. Tepley (1962), Hydromagnetic emissions, X-ray bursts, and electron bunches. 2. Theoretical interpretation, J. Geophys. Res. 67, 3335-3343.

Wentworth, R. C. (1964), Evidence for maximum production of hydromagnetic emissions above the afternoon hemisphere of the earth. 1. Extrapolation to the base of the ionosphere, J. Geophys. Res. 69, 2689-2698.

Wilson, C. R., and M. Sugiura (1961), Hydromagnetic interpretation of sudden commencements of magnetic storms. J. Geophys. Res. 66, 4097-4111.

(Paper 69D3-471) 